\title{
Local Stable or Unstable Regions in 2-Dimensional Chaotic Forms: Examples and Simulations
}

\author{
C.H. SKIADAS* \\ ManLab, Technical University of Crete, Greece
}

\begin{abstract}
We analyze 2-dimensional chaotic forms resulting from very simple systems based on two chaotic characteristics that is rotation and parallel movement or translation in geometric terms. Reflection is another alternative, along with rotation, for several interesting chaotic formations. Rotation and translation are very common types of movements in the world around us. Chaotic or non-chaotic forms arise from these two main generators. The rotation-translation chaotic case presented is based on the theory we analyzed in the book and in the paper ${ }^{\dagger}$. An overview of the chaotic flows in rotation-translation is given. There is observed the presence of chaos when discrete rotation-translation equation forms are introduced. In such cases the continuous equations analogue of the discrete cases is useful. Characteristic cases and illustrations of chaotic attractors and forms are analyzed and simulated. The analysis of chaotic forms and attractors of the models presented is given along with an exploration of the characteristic or equilibrium points. Applications in the fields of astronomy-astrophysics (galaxies), chaotic advection (the sink problem) and Von Karman streets are presented.
\end{abstract}

DOI: 10.12693/APhysPolA.124.1082

PACS: 95.10.Fh, 47.52.+j, 05.45.Pq, 98.62.-g, 47.32.C-, 47.32.C-, 47.32.Ef, 45.20.dc

\section{Introduction}

In this paper we explore important 2-dimensional chaotic cases resulting from two simple characteristics that is rotation or reflection and linear movement or translation in geometric terms. The chaotic attractors "locate" a plane by means of a space region of a planar form. A line is created by the intersection of two non-parallel planes. If these two planes are located by two interacting 2-dimensional chaotic attractors their interaction will form a line. A line movement could be the result of two interacting planar attractors. This problem appears in the study of coupled oscillations. The task here is to present and explore some basic plane chaotic forms appearing frequently in real life applications thus verifying some important characteristics of 2-dimensional chaotic forms. In the following the main findings from the chaotic literature are presented.

\section{Main chaotic findings}

From the model building point of view and according to the scientific developments in the non-linear and chaotic literature we can summarize the main findings as follows:

- Highly complicated forms that arise in chaotic attractors can be produced by relatively simple non-linear differential and difference equations (a first example is the model of May [1]).

\footnotetext{
*e-mail: skiadas@cmsim.net

${ }^{\dagger}$ See Refs. [6, 7].
}

- The main futures of chaotic forms and chaotic attractors can be produced by using equations including only few basic parameters the chaotic parameters (Lorenz [2] showed that a relatively simple chaotic model could express the basic chaotic behaviour of a more complicated case. See also the paper by Hénon [3]).

- Another very crucial point arises from the presence of chaotic forms in two-dimensional space because, based on the stability of chaotic forms, we can locate a plane.

- A straight line results as the intersection of two non-parallel planes so the two two-dimensional chaotic forms can locate a line movement.

- In several cases the difference equations' alternatives of the differential equations expressing a non-linear system, may provide and illustrate the hidden chaotic characteristics of the system.

- The most popular case is that of systems expressed by the differential logistic equation that provides non-chaotic forms whereas the difference equation alternative of the logistic one may give chaotic forms for a range of the chaotic parameter.

- Flows and especially chaotic flows are characterized by rotation or reflection and translation or transport. (A very popular model is proposed by Ikeda [4], whereas Aref [5] introduced a related theory to two-dimensional flows.)

- Many chaotic flows can be considered as being formed from very few but essential elements, which can be viewed, from a geometric point of view, as consisting of only two basic components, namely 
rotation in a specific plane and translation or transport in a specific direction.

\section{The related theory}

We present the basic elements from the parametric representation of a particle at place $\left(x_{n}, y_{n}\right)$ at a distance $r_{n}$ from the origin and rotation angle $\theta_{n}$. In polar coordinates it is given by

$$
x_{n}=r_{n} \cos \left(\theta_{n}\right), \quad y_{n}=r_{n} \sin \left(\theta_{n}\right),
$$

where $\theta_{n}$ is the rotation angle and $r_{n}=\sqrt{x_{n}^{2}+y_{n}^{2}}$.

If the system from step $n$ at time $t$ is rotated to step $n+1$ at time $t+1$ with an angle $\Delta \theta$, while $r_{n+1}=r_{n}$, the above equations yield

$$
\begin{aligned}
& x_{n+1}=r_{n} \cos \left(\theta_{n}+\Delta \theta\right), \\
& y_{n+1}=r_{n} \sin \left(\theta_{n}+\Delta \theta\right)
\end{aligned}
$$

and

$$
\begin{aligned}
& x_{n+1}=r_{n}\left[\cos \left(\theta_{n}\right) \cos (\Delta \theta)-\sin \left(\theta_{n}\right) \sin (\Delta \theta)\right], \\
& y_{n+1}=r_{n}\left[\cos \left(\theta_{n}\right) \sin (\Delta \theta)+\sin \left(\theta_{n}\right) \cos (\Delta \theta)\right] .
\end{aligned}
$$

The rotation map in difference equation form results by using the parametric equations of the circle

$$
\begin{aligned}
& x_{n+1}=x_{n} \cos (\Delta \theta)-y_{n} \sin (\Delta \theta), \\
& y_{n+1}=x_{n} \sin (\Delta \theta)+y_{n} \cos (\Delta \theta) .
\end{aligned}
$$

When rotation and transport (translation in geometric terms, equal to a quantity $a$ parallel to the $x$ axis) are considered, the movement of rotating particles following the last equations leads to the following rotation-translation difference equations:

$$
\begin{aligned}
& x_{n+1}=a+x_{n} \cos (\Delta \theta)-y_{n} \sin (\Delta \theta), \\
& y_{n+1}=x_{n} \sin (\Delta \theta)+y_{n} \cos (\Delta \theta) .
\end{aligned}
$$

The following iterative map represents a convenient form of the last set of difference equations:

$$
f_{n+1}=a+f_{n} \mathrm{e}^{\mathrm{i} \Delta \theta}, \quad f_{n}=x_{n}+i y_{n} .
$$

According to the theory developed in Refs. [6-8] we form the following differential equations analogue:

$$
\begin{aligned}
& \dot{x}=a+x[\cos (\Delta \theta)-1]-y \sin (\Delta \theta), \\
& \dot{y}=x \sin (\Delta \theta)+y[\cos (\Delta \theta)-1] .
\end{aligned}
$$

This set of differential equations is the continuous alternative of the discrete case presented by difference Eqs. (5). By applying the set of the two last coupled Eqs. (7) we can model trajectories or closed loops and compare these forms with their alternatives derived from the set of discrete Eqs. (5). Only the chaotic regions modeled by Eqs. (5) have no alternatives based on Eqs. (7), because the last set of two differential equations cannot model chaotic forms.

\section{Rotations under an inverse square law}

\subsection{Continuous case (differential equations)}

When the rotation angle is small $(\Delta \theta \ll 1)$ we can take the first order Taylor approximation for cos and sin and the last system of differential equations can be simplified to

$$
\dot{x}=a-y(\Delta \theta), \quad \dot{y}=x(\Delta \theta) .
$$

Combining both above equations

$$
\frac{\mathrm{d} y}{\mathrm{~d} x}=\frac{x \Delta \theta}{a-y \Delta \theta}
$$

and the equation

$$
a \mathrm{~d} y=(\Delta \theta) r \mathrm{~d} r,
$$

one can determine the form of the function $\Delta \theta$ during time. In classical mechanics the transverse component of the acceleration in a circular movement is expressed by the formula ( $c$ is a constant):

$$
r^{2} \dot{\theta}=c .
$$

From this relation, provided that $\Delta t=1$, we have

$$
\Delta \theta \approx \frac{c}{r^{2}} \text {. }
$$

A more general form of the formula $r^{2} \dot{\theta}=c$ appears in Taylor-Couette flows [7] where the angular velocity is of the form

$$
\dot{\theta}=k+\frac{c}{r^{2}} \text {. }
$$

However, Eq. (13) gives similar chaotic forms as the simpler form (12).

Using Eq. (12) for the rotation angle, the solution of Eq. (10) is

$$
c \ln (r)=a y+h
$$

and

$$
\frac{c}{2} \ln \left(x^{2}+y^{2}\right)=a y+h .
$$

According to $[6,7]$ the graphs for the rotation-translation are presented in Fig. 1A for the continuous case and Fig. 1B for the discrete case for various values of parameter $h(a=0.2, c=1)$. The similarities in the trajectories are obvious, whereas in the discrete case a chaotic bulge appear.

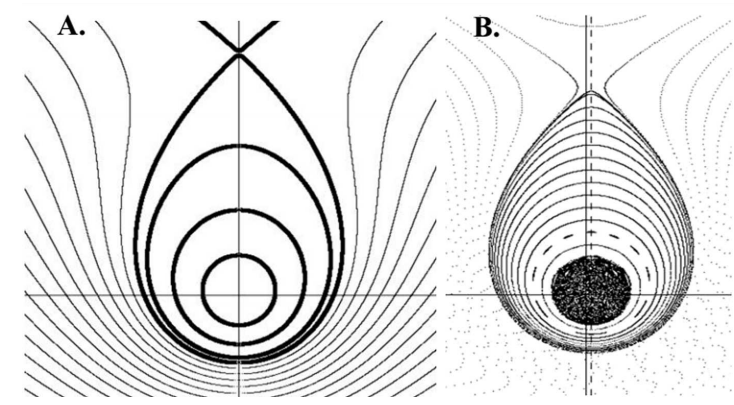

Fig. 1. Rotations under an inverse square law: A. continuous case, B. discrete case.

\subsection{Another form of the rotation-translation model: a family of ellipses}

A simple idea for expressing the rotation angle is coming from the relatively recent findings in astrophysics and more specifically the observation that the rotation speed 
(the radial rotation $u$ ) of the stars away from the rotation centre of the galaxies is relatively stable that it can be approximated by

$$
u=r \dot{\theta}=c .
$$

The solution of the differential Eq. (10) is

$$
a y+h=c r
$$

and

$$
c^{2}\left(x^{2}+y^{2}\right)=(a y+h)^{2} .
$$

This is the equation of a family of ellipses of the form $[6,7]$ :

$$
\left(\frac{x}{\sqrt{1-a^{2}}}\right)^{2}+\left(y-\frac{a h}{1-a^{2}}\right)^{2}=\frac{h^{2}}{\left(1-a^{2}\right)^{2}} .
$$

The resulting graphs are for the continuous (Fig. 2A) and discrete (Fig. 2B) case [6,7] (the symmetry axis is at $x=a / 2$. The parameters selected are $a=0.5$ and $c=1)$.

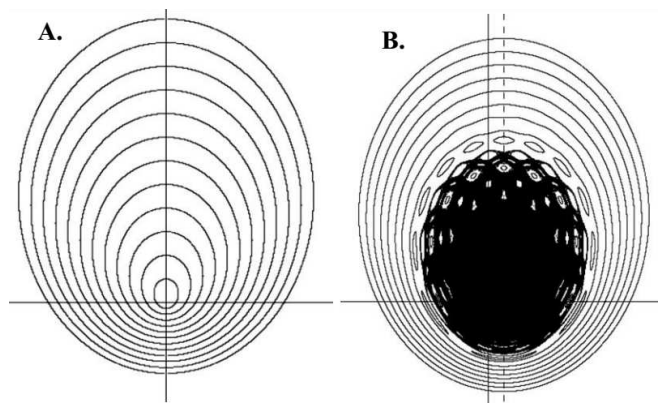

Fig. 2. A family of ellipses: A. continuous case, B. discrete case.

A very important property of the elliptic case is that the rotating particles remain in the elliptic paths even if high values for the parameter $h$ are selected. In the limit when the parameter $a$ approaches zero, the ellipses turn to be concentric circles. We thus see that in the case of a constant transverse velocity in a circular movement with translation the resulting trajectories follow elliptic paths and thus any particle remains on the corresponding path.

The particles do not fly to infinity as it can be in the first case presented earlier.

A system in the presence of a constant transverse velocity has a better ability to retain the particles into orbit than a system following the law of the classical central force. This finding is very essential for the case of rotating systems as are the galaxies. The previous case of elliptic trajectories makes the galactic system more stable. The chaotic elliptic forms keep their structure for long time periods. An important issue refers to the exploration of elliptic galaxies stability in an expanding universe. However, this could be the case of another study.

Of course, it remains a serious and yet unsolved problem. Such type galactic movements violate the classical theories related to the Newtonian dynamics.

However, it is now accepted by the majority of astrophysicists that a hidden and yet not observed mass is in the galactic objects termed as dark matter. From our analysis' point of view we do not need to make any speculations for the galactic dynamics or for the form or properties of the unknown dark matter.

We only use in our modeling the fact resulting from the astronomical observations that the transverse velocity of the particles or stars, in the case of a galaxy, in distances away from the center is almost stable.

\section{An open problem}

Find the direction of the principal axis of an elliptical galaxy regarding to the radial and transverse movement.

\section{Von Karman streets}

\subsection{A simulation of the Von Karman streets by using the reflection-translation theory}

The famous Von Karman chaotic phenomena [9] are presented in several formations, especially in the sea and are illustrated by photographs taken from space (satellite views).

The Von Karman phenomenon appears when in a fluid stream, fluid is flowing around an obstacle in the form of a cylinder (a vortex street develops behind a cylinder moving through fluid) or an island in the ocean. In the latter case the flow (fluid) lines or trajectories pass both sides of the island. To simplify the modeling we observe that a mirror image of the flow appears in both sides of the island or a reflection-like process appears. The translation parameter is added to keep the direction of the flow. In modeling the process the direction of flow is parallel to the $x$ axis.

The phenomenon can be modeled by using a reflection-translation equations set of the form

$$
\begin{aligned}
& x_{n+1}=a+b\left[x_{n} \cos (\Delta \theta)+y_{n} \sin (\Delta \theta)\right], \\
& y_{n+1}=b\left[x_{n} \sin (\Delta \theta)-y_{n} \cos (\Delta \theta)\right],
\end{aligned}
$$

where $a$ is the translation parameter and $b$ is the space contraction $(b<1)$ or expansion $(b>1)$ parameter. In the most cases $b=1$ is applied.

The rotation angle selected is of the form: $\Delta \theta=d / r^{k}$. $d, k$ are parameters and $k>0$. The rotation angle for the reflection-translation scheme must assure that the influence of the obstacle (island) is negligible at long distances, thus the parameter $k$ in the denominator should be large. The parameter $d$ accounts for the reflection and can be taken as $2 \pi$ or $\pi$.

\subsection{Numerical simulation}

The simulation starts from an original circle of rotating particles with $R=1$ at $(0 ; 0)$. The parameters selected are $d=2 \pi, a=1, k=3, t=10$. Behind the island the flow follows by forming chaotic forms or vortices. The distance between vortices (the frequency of vortex shedding) is equal to $a$.

Another point is to simulate the reflection-translation case. It can be achieved by a space expansion by means 


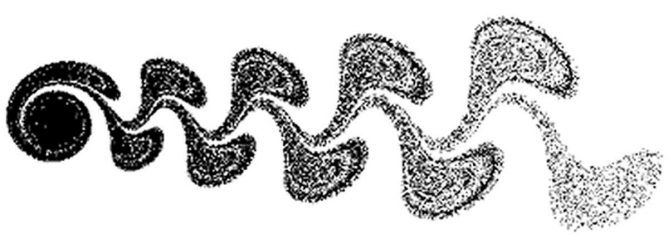

Fig. 3. Von Kármán vortex street simulation. Original circle of rotating particles $R=1$ at $(0,0), d=2 \pi, a=1$, $k=3, t=10$, and space expansion parameter $b=1.1$.

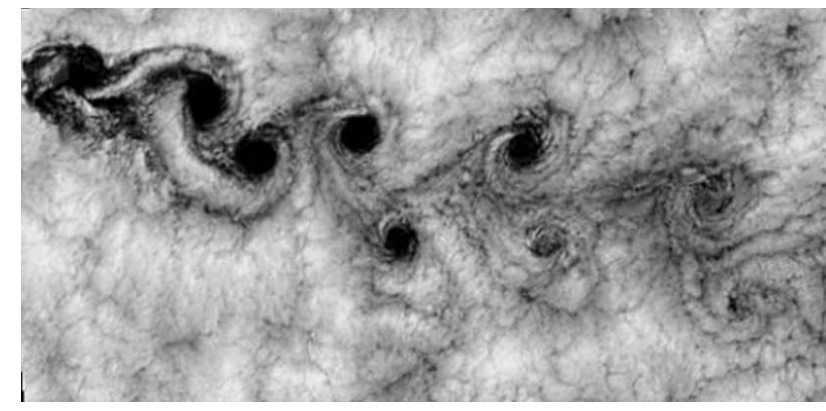

Fig. 4. Von Kármán vortex street off the Chilean coast near the Juan Fernandez Islands.

of a space expansion parameter $b=1.1$. The resulting form is illustrated in Fig. 3. The resulting chaotic forms slowly disappear under the space expansion process.

An example [6,9] that follows the later space expansion case is illustrated in Fig. 4. This is Landsat 7 image of clouds of the Chilean coast near the Juan Fernandez Islands (also known as the Robinson Crusoe Islands) on September 15, 1999. It is one of the classical Von Karman vortex streets pictures.

The simulation is quite representative of the phenomenon, although it is only a 2-dimensional modelling of a 3-dimensional case. The introduction of a third equation expressing the vertical movements to the $z$ direction would improve the simulation results.

\section{Chaotic advection}

\subsection{The sink problem}

Consider a circular bath with a sink at $(x, y)=(a, 0)$. The water inside the bath is rotating counterclockwise. A coloured fluid is injected in the periphery of the bath $[7,10]$. The question is to find the shape of the fluid filaments when we open the sink. The problem is analogous to a rotation with contraction for a parameter $b<1$. The rotation model is applied ( $a=0$ for a central sink):

$$
x_{n+1}=b\left[\left(x_{n}-a\right) \cos (\Delta \theta)-y_{n} \sin (\Delta \theta)\right] \text {, }
$$$$
y_{n+1}=b\left[\left(x_{n}-a\right) \sin (\Delta \theta)+y_{n} \cos (\Delta \theta)\right] \text {. }
$$

Assuming that the rotation angle follows an equation of the form:

$$
\Delta \theta=c+\frac{d}{r^{2}},
$$

where

$$
r=\sqrt{(x-a)^{2}+y^{2}},
$$

the fixed points of the map are given by the equation

$$
x^{2}+y^{2}=b^{2}\left[(x-a)^{2}+y^{2}\right]
$$

or, after transformation:

$$
\left(x+\frac{a b^{2}}{1-b^{2}}\right)^{2}+y^{2}=\left(\frac{a b}{1-b^{2}}\right)^{2} .
$$

Equation (23) represents a circle with radius $R=a b /(1-$ $\left.b^{2}\right)$ centred on

$$
(x, y)=\left(\frac{a b^{2}}{1-b^{2}}, 0\right),
$$

The flow is not symmetric. The coloured fluid starting from the outer periphery of the bath approaches the sink in few time periods as is illustrated in Fig. 5A. The parameters selected are $a=0.15, b=0.85, c=0$ and $d=0.1$. To simplify the process it is assumed that the coloured fluid is introduced in the periphery of the bath. The circular form of the original coloured line gradually is transformed to a chaotic attractor located at the sink's centre $(x, y)=(a, 0)$.

The space contraction is estimated from the Jacobian of the flow: $J=b^{2}$.

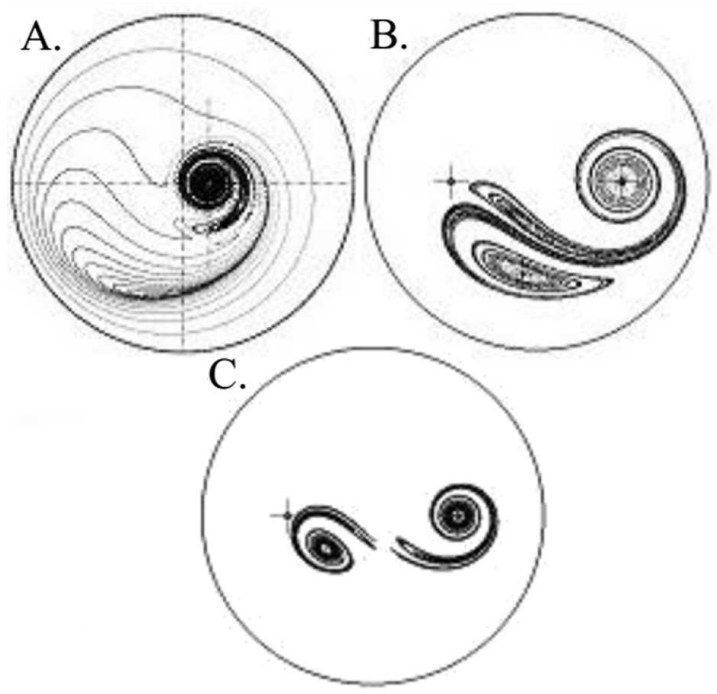

Fig. 5. Chaotic advection simulations: A. eccentric sink, B. two symmetric sinks $(d=3)$, C. two symmetric sinks $(d=1)$.

\subsection{Two symmetric sinks}

The governing equations are of the form

$$
\begin{aligned}
& x_{n+1}=a+b\left[\left(x_{n}-a\right) \cos (\Delta \theta)-y_{n} \sin (\Delta \theta)\right], \\
& y_{n+1}=b\left[\left(x_{n}-a\right) \sin (\Delta \theta)+y_{n} \cos (\Delta \theta)\right] .
\end{aligned}
$$

According to this system the flow is not stationary and there are jumps in the velocity field at each half pe$\operatorname{riod} T / 2$. In other words a particle located at $(-a, 0)$ appears at $(a, 0)$ the next time period.

The graph of Fig. 5B of a chaotic attractor illustrates the two-sink case for parameter values $a=1, b=0.8$, 
$c=\pi$ and $d=3$. Following the results presented in $[6,10]$ there are two main vortex forms counter-balancing each other. The first form is located in the right hand side sink at $(x, y)=(a, 0)$. The second vortex form is centered at $(x, y)=(a+2 a b \cos (\Delta \theta), 2 a b \sin (\Delta \theta))$, where $\Delta \theta=d /\left(4 a^{2}\right)$. The two main vortex forms can be separated when the parameter $d$ expressing the vortex strength is relatively small. This case is presented in the next Fig. $5 \mathrm{C}$. The parameter $d=1$ while the other parameters remain the same as in the previous case. Now the attractor is completely separated into two chaotic vortex forms (attractors).

\section{Conclusions}

We have given and explored several examples of two-dimensional (plane) chaotic flows.

The results support our main argument on the existence of several theoretical and real cases of plane chaotic forms in various natural phenomena.

The next step should be on finding theoretical, and then applied cases, where two plane chaotic forms interact, forming straight lines. After this step the movement and the stability of flows in these straight lines should be of particular importance.

\section{References}

[1] R.M. May, Nature 261, 459 (1976).

[2] E.N. Lorenz, J. Atmos. Sci. 20, 130 (1963).

[3] M. Hénon, C. Heiles, Astron. J. 69, 73 (1964).

[4] K. Ikeda, Opt. Commun. 30, 257 (1979).

[5] H. Aref, Ann. Rev. Fluid Mech. 15, 345 (1983).

[6] C.H. Skiadas, C. Skiadas, Chaotic Modelling and Simulation: Analysis of Chaotic Models, Attractors and Forms, CRC/Taylor and Francis, Boca Raton (FL) 2008.

[7] C.H. Skiadas, C. Skiadas, Int. J. Bifurcation Chaos 21, 3023 (2011).

[8] C.H. Skiadas, "Chaotic dynamics in simple rotationreflection models," in: Int. Workshop on Galaxies and Chaos: Theory and Observations, Academy of Athens, Astronomy Research Center, Athens 2002.

[9] C.H. Skiadas, in: Topics on Chaotic Systems, World Sci., Singapore 2009, p. 309.

[10] C.H. Skiadas, in: Recent Advances in Stochastic Modeling and Data Analysis, World Sci., Singapore, 2007, p. 287. 